\title{
DNA Repair: a Key Mechanism Stabilizing the Genome
}

DOI: $10.1134 / \mathrm{S} 0006297911010020$

This issue of Biokhimiya/Biochemistry (Moscow) presents reviews and experimental papers written by Russian and foreign authors that consider studies of DNA repair mechanisms and their role in maintaining the stability of the genome. Repair mechanisms responsible for correcting DNA damages provide for the safety of genetic information. Factors negatively influencing the stability of the human genome include mutagens, carcinogens, UV and ionizing radiation, and various chemical substances, e.g. products of tobacco smoke and of incomplete combustion of fuels. The increasing level of these factors in the environment increases the frequency of oncologic and cardiovascular diseases and results in premature aging.

The action of many exogenous and endogenous toxic agents directly or indirectly induces genotoxic stress accompanied by damage of genomic DNA. During evolution some refined and partially overlapping systems have been developed that are capable of correcting the majority of changes in genetic "texts" of cells by elimination of DNA damage. These systems include direct repair, base excision repair, nucleotide excision repair, repair of double-strand breaks in DNA (homologous recombination and non-homologous end joining), and mismatch repair. This issue of Biokhimiya/Biochemistry (Moscow) contains papers about action mechanisms of some of these systems and their regulation, and also papers concerning the replicative synthesis of DNA on damaged templates.

Studies on mechanisms of DNA repair and replication and also on regulation of these processes are now a cutting edge the global science because this field of molecular biology is directly connected with both searches for approaches promoting the survival of organisms and for optimal treatments of cancer and other human diseases. Damage of DNA is the major way in the treatment of malignant tumors by chemotherapeutic drugs. Ionizing radiation is also widely used. The therapeutic effect of these agents depends on activities of DNA repair systems, because they eliminate DNA damage and this lowers the efficiency of chemo- and radiotherapy. Therefore, studies on the DNA repair systems and determination of their status in the cells of healthy subjects and patients are very important for clinical practice.

This issue contains contributions of leading researchers in the field of DNA repair mechanisms from different countries, for example Eugenia Dogliotti (Department of Environment and Primary Prevention, Istituto Superiore di Sanità, Italy) and Kaoru Sugasawa (Biosignal Research Center, Organization of Advanced Science and Technology, Kobe University, Japan). Other authors of works presented in this issue are graduates of the Novosibirsk State University who are successfully working in other countries: Grigory Dianov (Gray Institute for Radiation Oncology and Biology, University of Oxford, Great Britain), Alexander Mazin (Department of Biochemistry and Molecular Biology, Drexel University College of Medicine, USA), and also scientists from St. Petersburg working now in USA - Yury Pavlov and Pauline Shcherbakova (Eppley Institute for Research in Cancer and Allied Diseases, University of Nebraska Medical Center, USA). Many other papers are presented by leading researchers working in institutes of the Russian Academy of Sciences.

Note that studies on processes of DNA repair and practical aspects associated with creation of inhibitors and activators of these systems are now successfully studied in Russia. Thus, this is one of the leading directions of investigations in the Institute of Chemical Biology and Fundamental Medicine of the Siberian Branch of the Russian Academy of Sciences. The papers and reviews presented in this issue of the journal will allow the reader to look through the latest findings in the field and the most urgent problems in studies on DNA repair and replication.

Editor of Special Issue, Doctor of Chemistry, Corresponding Member of the Russian Academy of Sciences O. I. Lavrik 